\title{
Affordable Phenotyping of Winter Wheat under Field and Controlled Conditions for Drought Tolerance
}

\author{
Dhananjay Kumar ${ }^{1}$, Sandeep Kushwaha ${ }^{1,2}$, Chiara Delvento ${ }^{1,+}{ }^{6}$, Žilvinas Liatukas ${ }^{3}$, \\ Vivekanand Vivekanand ${ }^{4}$, Jan T. Svensson ${ }^{5}$, Tina Henriksson ${ }^{6}$, Gintaras Brazauskas ${ }^{3}$ and \\ Aakash Chawade $1, *$ (i) \\ 1 Department of Plant Breeding, Swedish University of Agricultural Sciences (SLU), Box 101, \\ SE-230 53 Alnarp, Sweden; dhananjay.kumar@slu.se (D.K.); sandeep@niab.org.in (S.K.); \\ chiara.delvento@uniba.it (C.D.) \\ 2 National Institute of Animal Biotechnology, Hyderabad 500032, India \\ 3 Institute of Agriculture, Lithuanian Research Centre for Agriculture and Forestry, \\ LT-583 44 Akademija, Lithuania; zilvinas.liatukas@lammc.lt (Ž.L.); gintaras.brazauskas@lammc.lt (G.B.) \\ 4 Centre for Energy and Environment, Malaviya National Institute of Technology, Jaipur 302017, India; \\ vivekanand.cee@mnit.ac.in \\ $5 \quad$ Nordic Genetic Resource Center, Box 41, SE-230 53 Alnarp, Sweden; jan.svensson@nordgen.org \\ 6 Lantmännen Lantbruk, SE-268 31 Svalöv, Sweden; tina.henriksson@lantmannen.com \\ * Correspondence: aakash.chawade@slu.se; Tel.: +46-40-415-328 \\ + Current address: Department of Soil, Plant and Food Science, University of Bari "Aldo Moro", \\ 70126 Bari, Italy.
}

Received: 9 May 2020; Accepted: 15 June 2020; Published: 19 June 2020

\begin{abstract}
Drought stress is one of the key plant stresses reducing grain yield in cereal crops worldwide. Although it is not a breeding target in Northern Europe, the changing climate and the drought of 2018 have increased its significance in the region. A key challenge, therefore, is to identify novel germplasm with higher drought tolerance, a task that will require continuous characterization of a large number of genotypes. The aim of this work was to assess if phenotyping systems with low-cost consumer-grade digital cameras can be used to characterize germplasm for drought tolerance. To achieve this goal, we built a proximal phenotyping cart mounted with digital cameras and evaluated it by characterizing 142 winter wheat genotypes for drought tolerance under field conditions. The same genotypes were additionally characterized for seedling stage traits by imaging under controlled growth conditions. The analysis revealed that under field conditions, plant biomass, relative growth rates, and Normalized Difference Vegetation Index (NDVI) from different growth stages estimated by imaging were significantly correlated to drought tolerance. Under controlled growth conditions, root count at the seedling stage evaluated by imaging was significantly correlated to adult plant drought tolerance observed in the field. Random forest models were trained by integrating measurements from field and controlled conditions and revealed that plant biomass and relative growth rates at key plant growth stages are important predictors of drought tolerance. Thus, based on the results, it can be concluded that the consumer-grade cameras can be key components of affordable automated phenotyping systems to accelerate pre-breeding for drought tolerance.
\end{abstract}

Keywords: wheat; drought; machine learning; affordable phenotyping

\section{Introduction}

Meeting the food demands for the growing world population is a challenging task for farmers, scientists, and policymakers. Wheat is one of the most widely grown and essential crops among cereals and contributes almost $20 \%$ of the total energy requirement of human food consumption [1]. 
However, improvement in wheat production is seen at a rate of approximately $0.9 \%$ per year, but this is far slower than the rates required to double production by 2050 [2]. Thus, to ensure food security, there is an urgent need to develop germplasm adapted to the changing climate [3]. A considerable impact of climate change is seen on the magnitude of rainfall, crop cycle, and crop productivity. Frequent droughts and water shortages have caused severe problems throughout the globe, resulting in significant yield losses. The inter-annual variability in weather conditions is seen in European countries [4,5]. The key challenges for sustainable wheat production in the Nordic region are natural variability in climate and global warming. The temperature rise has been noted at the rate of $0.05^{\circ} \mathrm{C}$ per decade from 1861 to 2000 throughout the globe. However, in the Baltic Sea basin, it has been noted to increase by $0.08{ }^{\circ} \mathrm{C}$ per decade. This temperature rise has a considerable impact on annual precipitation in the Baltic Sea region and affects the average wheat production [6,7].

For the development of drought-tolerant varieties, different types of soil and plant environmental conditions along with diverse genotypes can be used to generate highly diverse water deficit scenarios [8]. Because traits of interest are context-dependent, we need biometric tools and mathematical models to measure and integrate multiple overlapping mechanisms with high-throughput phenotyping platforms. Automated phenotyping is now a bottleneck in advancing crop yields $[9,10]$. The various platforms for phenotyping such as proximal sensing carts [11], field-scanners systems [12], and unmanned aerial systems [13] have earlier been implemented for field study. These systems can be mounted with various types of sensors, such as digital cameras [14] and multispectral cameras [13]. There are, however, immense challenges in efficiently estimating agronomic traits due to varying illumination and fluctuating weather conditions. Sensor-based phenotyping was used to study various morphological traits correlated with drought tolerance. Imaging-based plant biomass and relative growth rates (RGR) from the field correlated well with drought tolerance and can indicate early stress symptoms if the growth patterns differ from expected. It was earlier shown that RGR in early growth stages is positively correlated to drought tolerance in wheat under water deficit [15]. Simane et al. [16] found that the drought-tolerant genotypes had higher RGR under optimal conditions and low RGR under moisture stress, while the drought-susceptible genotypes showed the opposite trend. Normalized Difference Vegetation Index (NDVI) measurements also correlated with drought with the maximum correlation at the stem elongation stage, thus making it a suitable proxy estimate for drought stress. NDVI was previously shown to be an effective indicator of plant response to drought stress [17,18], and thus several quantitative trait loci (QTL) for NDVI were also identified [18]. Liu et al. [19] defined a threshold for NDVI below which the plants were considered to be under drought stress.

Machine learning tools such as random forests and support vector machines are frequently used during feature identification for stress phenotyping. These methods can be used for identification, classification, quantification, and prediction of various biotic and abiotic stress traits along with the ability to identify a hierarchy of features and generalized trends $[20,21]$. Clustering of data aims to group and select other traits of interest in such a way that the relation between traits should be maximal if they belong to the same group and vice versa [22]. The time series clustering analysis is used to measure the distance between genotypes. Shape-based measurements derived from the K-shape algorithm were used in a previous study [23,24]. Few reports attempted to use proximal phenotyping and unmanned aerial vehicles (UAV) phenotyping for multiple-trait measurement as well as analyze and evaluate dynamics and development trends of phenotypic traits among large-scale genotypes in the field [25-29]. In the current study, we investigated how the evaluation of drought tolerance in wheat can be estimated by affordable phenotyping using consumer-grade cameras and the barebone phenotyping system under both controlled and field conditions. 


\section{Material and Methods}

\subsection{Plant Material, Phenocart, and Phenotyping in the Field}

In this work, 142 winter wheat genotypes from the Nordic Genetic Resource Center (NordGen) were selected for screening for drought tolerance. The field trial was carried out in 2017-2018 in the field in southern Sweden and 2018-2019 in Akademija, Lithuania. The weather was hot and dry during both years and thus was an optimal environment to investigate drought stress. Three control genotypes were used in the trial in Sweden, and 2 replications and 10 local genotypes as controls were used in Lithuania. The field trial was repeated in 2018-2019 in Sweden with 2 replications and 10 local genotypes as controls, but there was no drought that year.

The phenocart was assembled based on the instructions provided by Crain et al. [29] with modifications. The dimensions (length: $2.5 \mathrm{~m}$, height: $3.5 \mathrm{~m}$, and width: $1.5 \mathrm{~m}$ ), and the width of the phenocart we built can be adjusted according to the row spacing and the plot width (Figure 1). Five different sensors can be mounted on the phenocart. A metal rod was mounted to the vertical outer frame to support all sensors attached to it. In the current study, as our focus was phenotyping just a few hundred plots, we chose a manually operated phenocart that was adequate to cover all plots within a few hours of fieldwork. All sensor modules attached to the phenocart were operated in the manual mode.

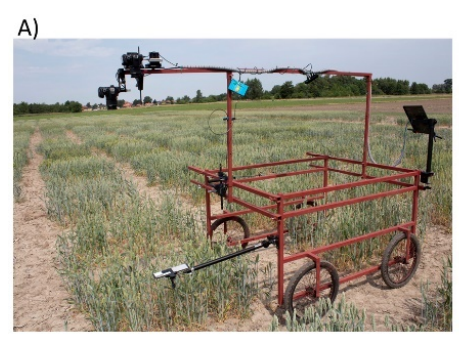

B)

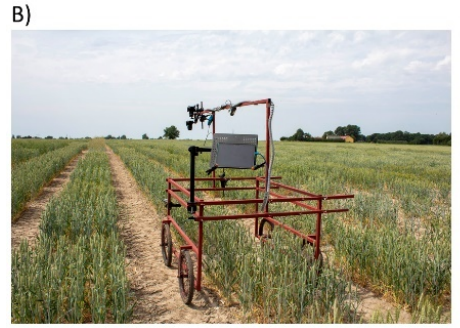

C)

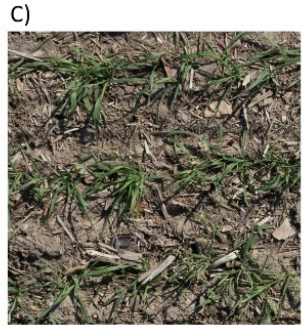

(1)

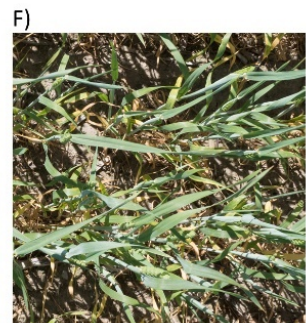

D)

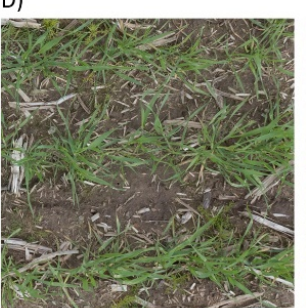

G)

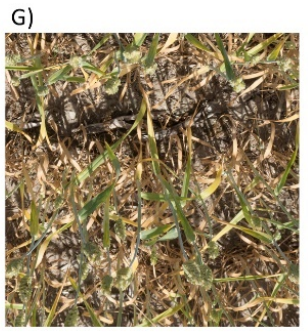

E)

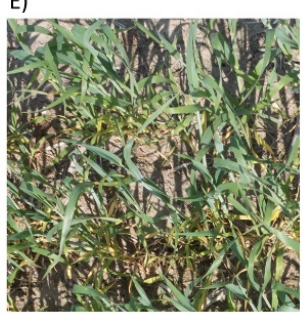

H)

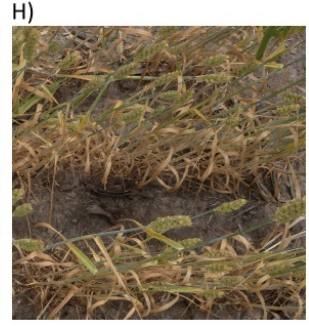

Figure 1. Phenocart and representative Red-green-blue (RGB) images of winter wheat at various growth stages. (A) and (B) Phenocart with a sensor bar, computer, various sensors, and a grey card. Representative RGB images at various growth stages: (C) seedling (Z14-19), (D) tillering (Z20-30), (E) stem elongation (Z30-39), (F) booting (Z40-49), and grain development stages (Z61-85) (G) GD1 and (H) GD2.

For visual imaging, we used two consumer-grade Digital Single Lens Reflectance (DSLR) cameras (Canon 1300d, Canon, USA), of which one camera was converted for NDVI imaging (Life Pixel Infrared, USA) capturing light spectrum in blue, green (approximately 400-600 nm), and near-infrared (approximately $700-800 \mathrm{~nm}$ ) range. Both DSLR cameras were triggered by the open-source software digiCamControl with manual settings leaving ISO and shutter speed to auto, allowing even exposure in all images. Each image also included a gray card (Electra, Photax P. Arvidsson Foto AB, Sweden), which was later used for exposure correction as described under the subsection "image processing". Visual or red-green-blue (RGB) imaging was done at six Zadoks growth stages [30], namely during seedling (Z14-19), tillering (Z20-30), stem elongation (Z30-39), booting (Z40-49), and twice during grain development stages (GD1 and GD2) (Z61-85) (Figure 1). NDVI imaging was done at booting, GD1 and GD2 stages. All generated data were tested for outliers prior to statistical analysis. 


\subsection{Plant Height and Drought Scoring}

Plant height was measured manually with a ruler at maturity. The height was measured from the ground to the tip of the spike of plants. For each plot, three measurements were made per plot, from the middle of the plots (mid-plot), and the two corners at 180 degrees. Each plot was given a unique plot number so that height measurements would be associated with the correct plot. Drought scoring of individual genotypes was performed from an average of all plants in a plot at the GD1 and GD2 growth stages. The drought scoring was based on the Standard Evaluation System for drought score [31]. The genotypes were scored on a linear scale of $0-9$ by visual inspection, where genotypes with no symptoms received 0 , slight leaf rolling and drying received scores from 1-3, moderate rolling and drying received scores from 4-6, more than two-third drying received scores from $7-8$, and those with dead plants received 9.

\subsection{Shoot and Root Phenotyping under Controlled Conditions}

Experiments under controlled climatic conditions were done in the controlled growth facility Biotron at The Swedish University of Agricultural Sciences (SLU), Alnarp, Sweden. The same genotypes studied in the field were grown in the Biotron at $23{ }^{\circ} \mathrm{C} / 19{ }^{\circ} \mathrm{C}$ day/night temperature with a $16 \mathrm{~h}$ photoperiod and a light intensity of $380 \mu \mathrm{mol} \mathrm{m}^{-2} \mathrm{~s}^{-1}$. Drought treatment under controlled conditions was carried out on plants grown in small pots $(8 \times 8 \times 8 \mathrm{~cm})$ containing peat substrate Blomjord Exclusive (Emmaljunga Torvmull AB, Sweden). Wheat seeds were cold stratified at $4{ }^{\circ} \mathrm{C}$ for $48 \mathrm{~h}$ for uniform germination. Two germinating seedlings were sown per pot, and the pots were arranged in augmented block design with six blocks and 34 genotypes including four checks in each block. Plants were phenotyped for shoot growth at 10 and 18 days after sowing (DAS). Thereafter, plants were exposed to drought stress for six days and phenotyped again. The entire experiment was repeated twice.

Shoot phenotyping was done as described in an earlier study [32] with some modifications. Briefly, LED lights with a color temperature of $5500 \mathrm{~K}$ were placed on both sides of the plant at an inclined angle, illuminating both the plant and the background. Root imaging was done in a well-lit growth chamber, as described earlier by Thomas et al. [33]. A blue marker was placed just beside the roots to aid in the framing of images. Imaging of both roots and shoots was performed with digital single-lens reflex (DSLR) cameras (Canon 1300D, Canon, USA) and the $18-55 \mathrm{~mm}$ kit lens. For roots, the camera was mounted on a Kaiser stand $40 \mathrm{~cm}$ above the root surface. For reading the QR code of the sample, a webcam (Logitech International S.A., USA) was placed just beside the root surface. The QR code containing desired metadata such as the cultivar name, replicate number, and treatment was generated with Bytescout barcode generator (https://www.bytescout.com) and printed on self-adhesive labels with a custom R script. QR code was set up to be read by the webcam placed upfront of the root paper and connected by a software called bcWebCam (http://www.bcwebcam.de). Thus, during imaging, all metadata information from the QR code was automatically transferred from bcWebCam (QS QualitySoft GmbH, Hamburg, Germany) to the software digiCamControl [34].

\subsection{Image Processing and Analysis}

RGB and near-infrared (NIR) images from the field were manually adjusted for white balance and exposure using the grey card included in each image; thereafter, the images were cropped to an even size to only retain the plant area using the open-source software RawTherapee v5.5 [35]. Biomass estimates were obtained from the RGB images from both the field and the controlled conditions and NDVI measurements from field NIR images using PlantCV [36] using the analysis pipeline for RGB images described earlier [32]. The analysis pipeline led to removal of soil from the field pictures and background from the pictures from the controlled conditions. For NIR images, NDVI was first estimated, and thereafter only those pixels were retained with values above 0.6 thereby removing soil and other debris from the images. Root images were analyzed with RootNav software following 
developer instructions [37]. Phenotypic data obtained from the controlled conditions with augmented design was corrected using the Agricolae package [38] in R [39].

\subsection{Random Forest}

The $\mathrm{R}$ package Caret was used to train Random Forest (RF) models for predicting drought tolerance based on plant growth estimates obtained on winter wheat genotypes from the field and under controlled conditions. From the field, growth estimates obtained from images from RGB camera and modified NDVI camera were used in addition to manually measured plant height. From the biotron, plant growth estimates from RGB imaging of roots and shoots were used for model training. Parameters for model training were tuned using the function trainControl in Caret. Ten-fold cross-validation repeated three times (repeats $=3$ ) was performed with the repeatedcv method, number of trees (ntree) 1000, and five different values (tuneLength 5) were tried for number of variables available for each split (mtry). Samples belonging to the same genotype were not selected as part of training and test set at the same time. The models were trained against the average drought scores obtained from the growth stages GD1 and GD2. Based on tuning, mtry with value of 18 was chosen as it had the lowest root-mean-square error (RMSE), and ntree was set to 1000 as the prediction accuracy did not improve further with more trees.

\section{Results}

\subsection{Phenotypic Characterization under Field Conditions}

The frequency distributions of the three traits, namely drought tolerance at GD1 and GD2 and plant height showed approximately normal distributions, while no variation was seen in the flag leaf angle (Figure 2). Drought scores were distributed in the range of 4-6 during GD1 ( $\bar{x} 5.4 \pm 1.2$ ) and $5-8(\bar{x} 5.8 \pm 1.5)$ during the GD2 stage (Table S1). The correlation between the two drought scores was 0.5, and that of the drought scores from the field trials in Sweden and Lithuania was 0.19. Phenotypic variation was also observed in plant height $(\bar{x} 80.4 \pm 1.2)$. Plant biomass was estimated from RGB imaging at six growth stages (seedling to grain development), and phenotypic variation was observed among genotypes across timepoints (Figure 3). Relative growth rates (RGR) between any two consecutive timepoints were measured from the plant biomass with the method described earlier [32,40]. RGR of the first two measured timepoints (from seedling to tillering) is known as early vigor and varies considerably among the evaluated genotypes (Figure 4). RGR from tillering to stem elongation (RGR.Til_SE) was the highest, and RGR from booting to GD1 was the lowest (RGR.Boot_GD1) among comparisons. NDVI measurements were estimated at booting and the two grain development stages. At booting, NDVI measurements $(0.32 \pm 0.13)$ indicated plant stress as the values were much lower than what is expected from healthy plants, and the values further decreased at the later two timepoints (Figure 5).
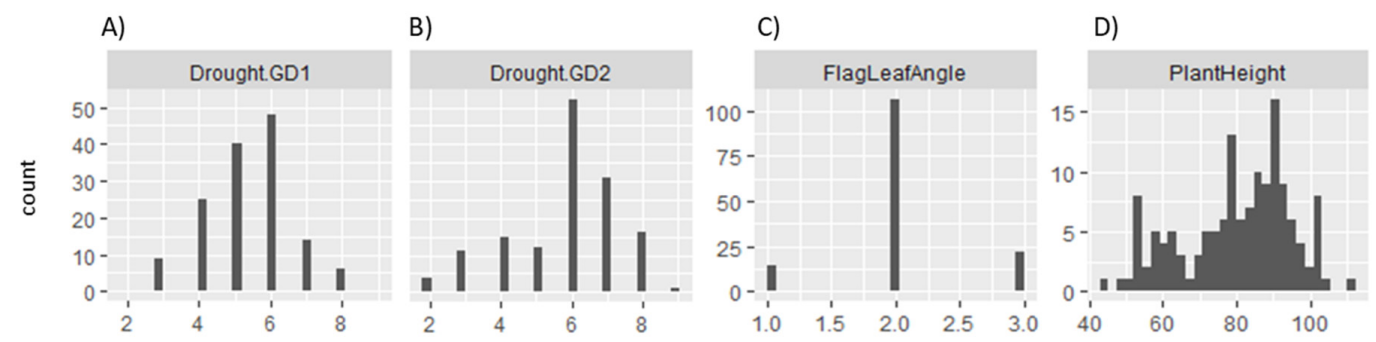

Figure 2. The frequency distributions for drought tolerance at heading and ripening stages, flag leaf angle, and plant height of the winter wheat germplasm (A-D). X-axis: the range for the obtained measurements in the respective units for each trait; Drought at two grain development stages (GD1 and GD2) (1-9), Flag leaf angle (1-3), and plant height (cm); Y-axis: the number of genotypes in each bin in that range. 


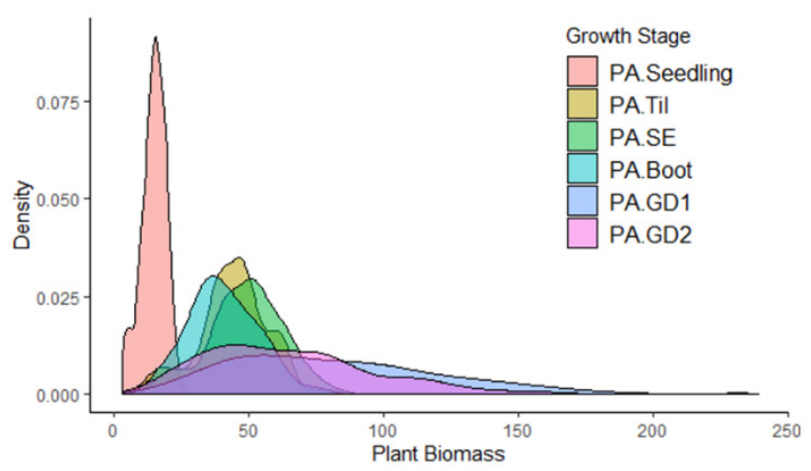

Figure 3. Density plots of plant area/biomass (PA) estimated by RGB imaging across six growth stagesseedling, tillering (Til), stem elongation (SE), booting (Boot), and grain development 1 and 2 (GD1 and GD2).

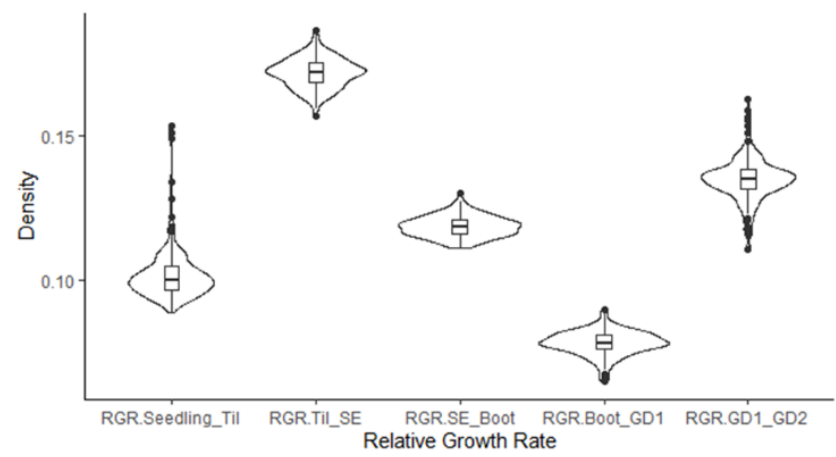

Figure 4. Relative growth rates (RGR) of genotypes between consecutive timepoints at six growth stagesseedling, tillering (Til), stem elongation (SE), booting (Boot), and grain development 1 and 2 (GD1 and GD2).

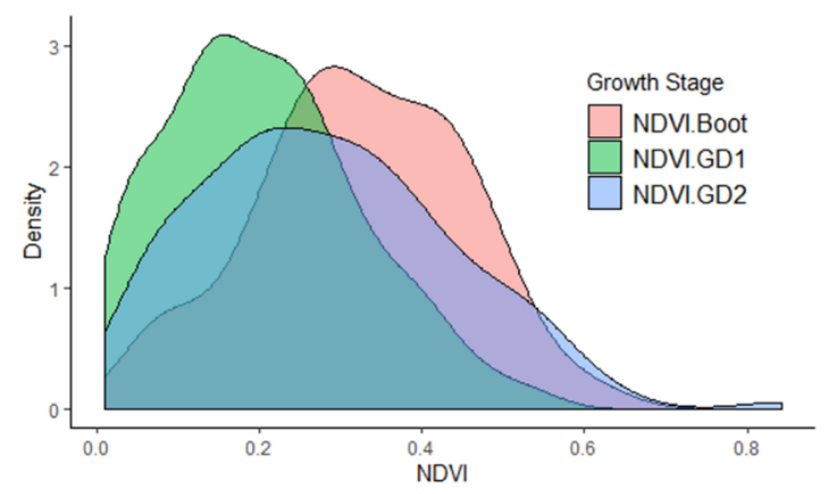

Figure 5. Density plots of Normalized Difference Vegetation Index (NDVI) obtained by imaging at three growth stages-booting (Boot) and grain development 1 and 2 (GD1 and GD2).

To further explore the response of the genotypes to drought stress over time, genotypes were clustered by K-means clustering into six groups based on their biomass estimated by RGB imaging at six timepoints in the field (Figure 6). Group 1 genotypes displayed the most dynamic growth pattern, while Group 6 genotypes showed the least. Group 1 genotypes were also the tallest, while Group 6 the shortest (Figure 7). A similar contrasting pattern can be seen in the comparison of these two groups for drought tolerance. Group 4 and 5 genotypes displayed moderate dynamic growth over the growth season (Figure 6) and were also found to have a higher tolerance to drought (Figure 7). Group 3 genotypes were the most drought-sensitive, while Group 6 genotypes had the most variation in drought tolerance (Figure 7). NDVI measured in booting and grain development stages was higher in drought-tolerant genotypes in Groups 1 and 4. For traits measured the seedling stage under controlled conditions, significant differences among the six groups were observed for root count 
and biomass of plants 18 DAS (GHPA18d), whereas drought tolerance at the seedling stage was not significantly different in the six groups (Figure 7).
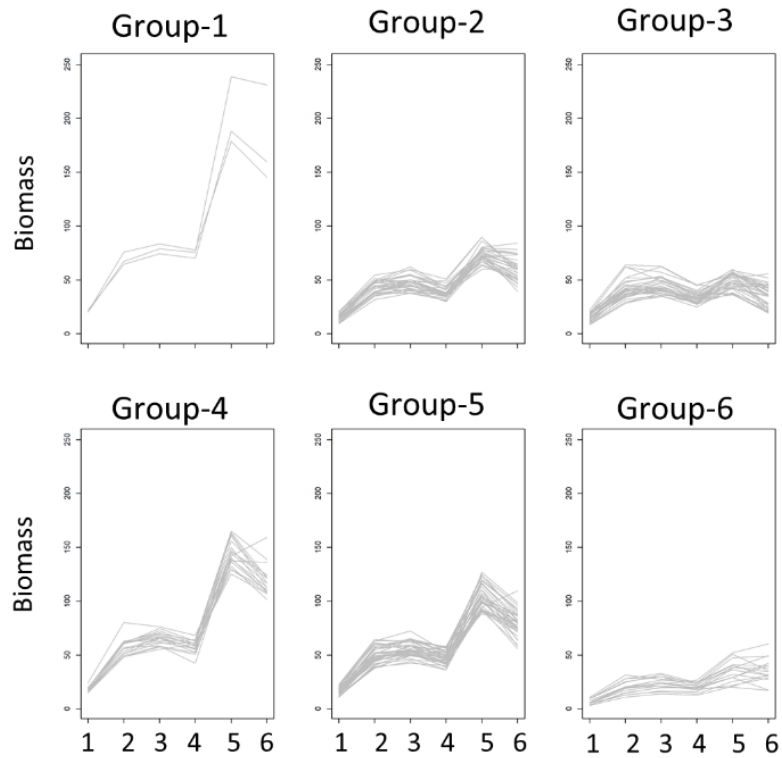

Figure 6. Plant growth pattern from tillering to grain development. Genotypes clustered by K-Means clustering based on their growth dimensions forming six groups. The Y-axis represents biomass and the X-axis represents six different growth stages (1) PA.Seedling, (2) PA.Til, (3) PA.SE, (4) PA.Boot, (5) PA.GD1, and (6) PA.GD2.
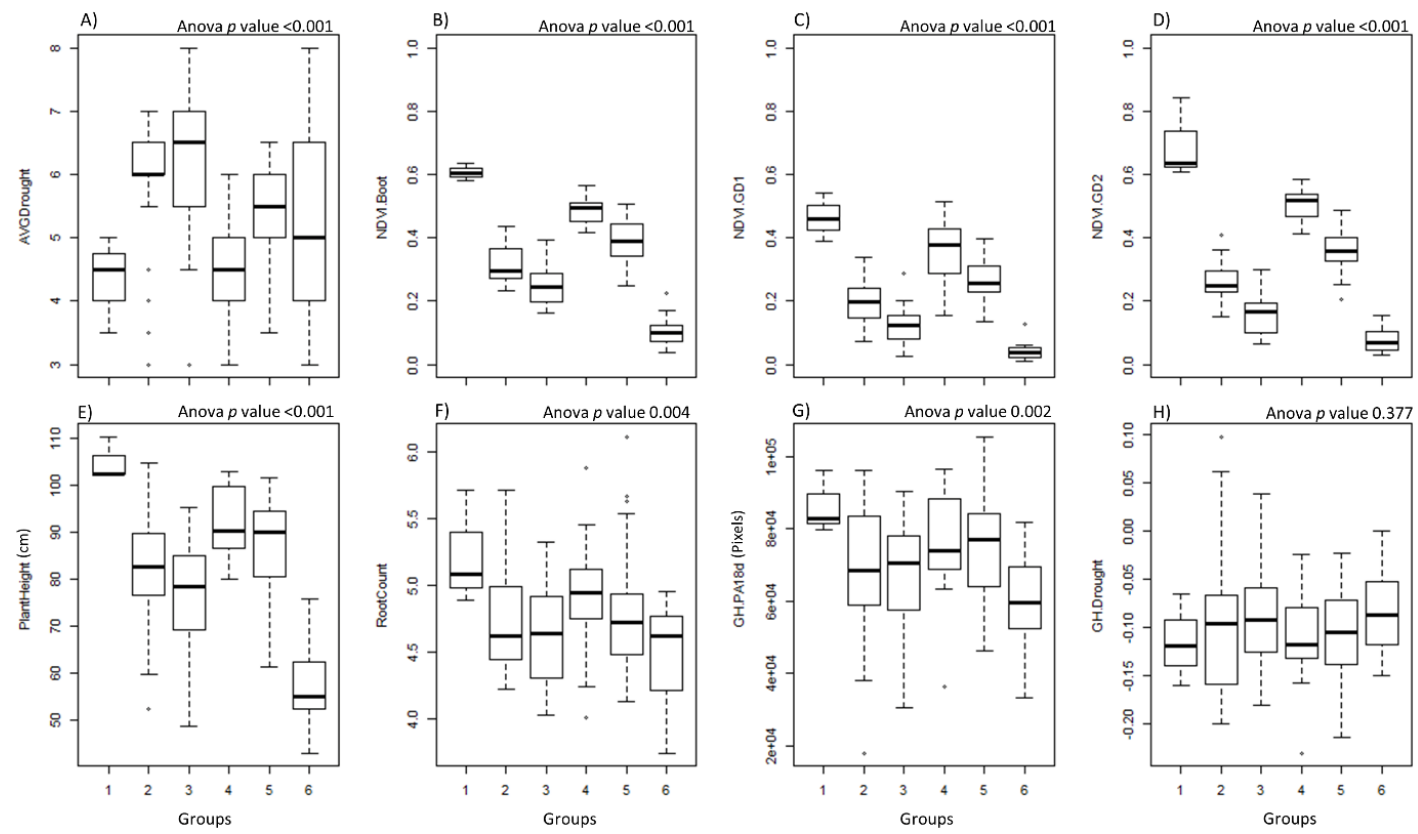

Figure 7. Analysis of variance for selected traits among genotypes belonging to the six groups formed by K-means clustering. (A) AVDrought: Average drought; (B) NDVI.Boot: NDVI at booting stage; (C) NDVI.GD1: NDVI at grain development stage 1; (D) NDVI.GD2: NDVI at grain development stage 2; (E) Plant height; (F) Root count; (G) GHPA18d: greenhouse plant area at 18 days; (H) GH.Drought; greenhouse drought.

\subsection{Phenotypic Characterization under Controlled Growth Conditions}

Early vigor trait was estimated under controlled conditions by imaging plants at 10 and 18 DAS showing normal distribution (Figure 8). The plants were thereafter drought-treated for six days and 
phenotyped (Figure 8). Drought stress analysis by imaging revealed NGB6713, NGB8946, NGB344 as the top three drought-tolerant genotypes at the seedling stage under controlled conditions. Root phenotyping under controlled conditions was performed for estimating eight different root traits, and phenotypic variation was observed for all eight traits (Figure 8). Genotypes with the longest roots at the seedling stage were NGB13446, NGB23349, NGB6700, while those with the most number of roots were NGB7183, NGB18, NGB12.
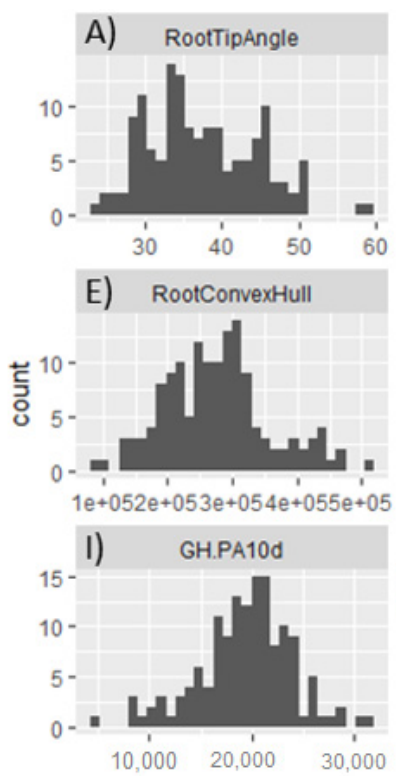

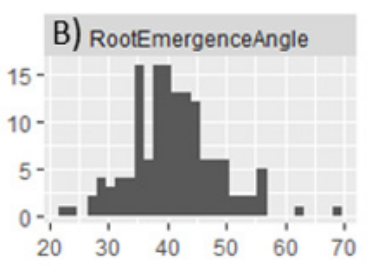

F) RootMaxWidth
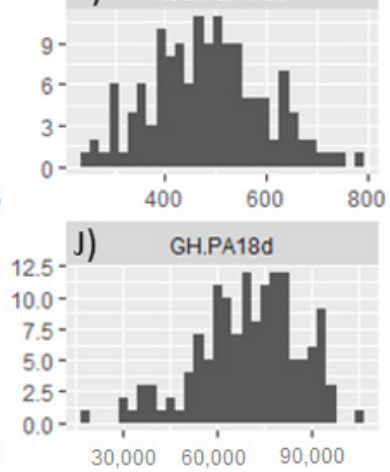
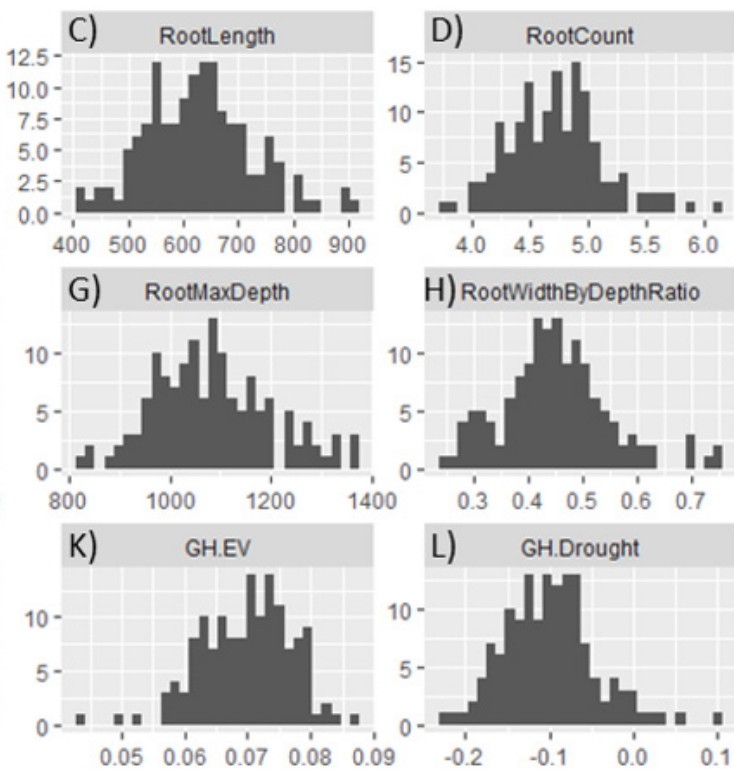

Figure 8. Frequency distribution for root and shoot traits measured under controlled conditions (A-L). X-axis: range for the obtained measurements in the respective units for each trait; Y-axis: the number of genotypes in each bin in that range. GH.EV: Greenhouse early vigour; GH.PA10: greenhouse plant area at 10 days; GH.PA18d: greenhouse plant area at 18 days.

\subsection{Correlation Analysis}

Spearman's rank correlation coefficient analysis was performed to estimate the degree of phenotypic correlation among all traits measured under field and controlled conditions (Figure 9). Under the field conditions, the correlation between plant biomass estimated at individual timepoints and the drought scores at GD1 and GD2 increased with the plant growth stage. Significant correlations were observed between drought at GD1 and plant biomass at all stages starting from tillering (Figure 9). The highest negative correlation was seen between the plant biomass at GD2 (PA.GD2) and drought score at GD1 (Drought) $(r-0.64)$. Significant correlations were observed among shoot biomass measured under controlled and field conditions. Correlation estimates were also obtained for relative growth rates (RGR) between any two given timepoints and drought. The least correlation was between RGR.Seedling_Til and Drought.GD1 $(r-0.15)$, and the highest correlation was between RGR.Til_GD2 and AVGDrought $(r-0.63)$. Correlation between NDVI at booting (NDVI.Boot) and Drought.GD1 was the highest $(r-0.52)$, and the NDVI correlation was overall slightly lower than what was observed from the traits obtained from RGB imaging and drought.

Root traits measured under controlled conditions displayed low to moderate correlations to drought scores from field conditions. RootTipAngle ( $r$ 0.11), RootCount $(r-0.21)$, RootMaxWidth $(r 0.10)$, and RootWidthByDepthRatio $(r 0.16)$ had moderate correlation with the field drought scores (AVGDrought), while the RootLength was interestingly not correlated to AVGDrought ( $r$ 0.08). Shoot biomass, shoot early vigor and seedling drought stress under controlled conditions were not correlated to field drought scores (Figure 9, Table S2). 


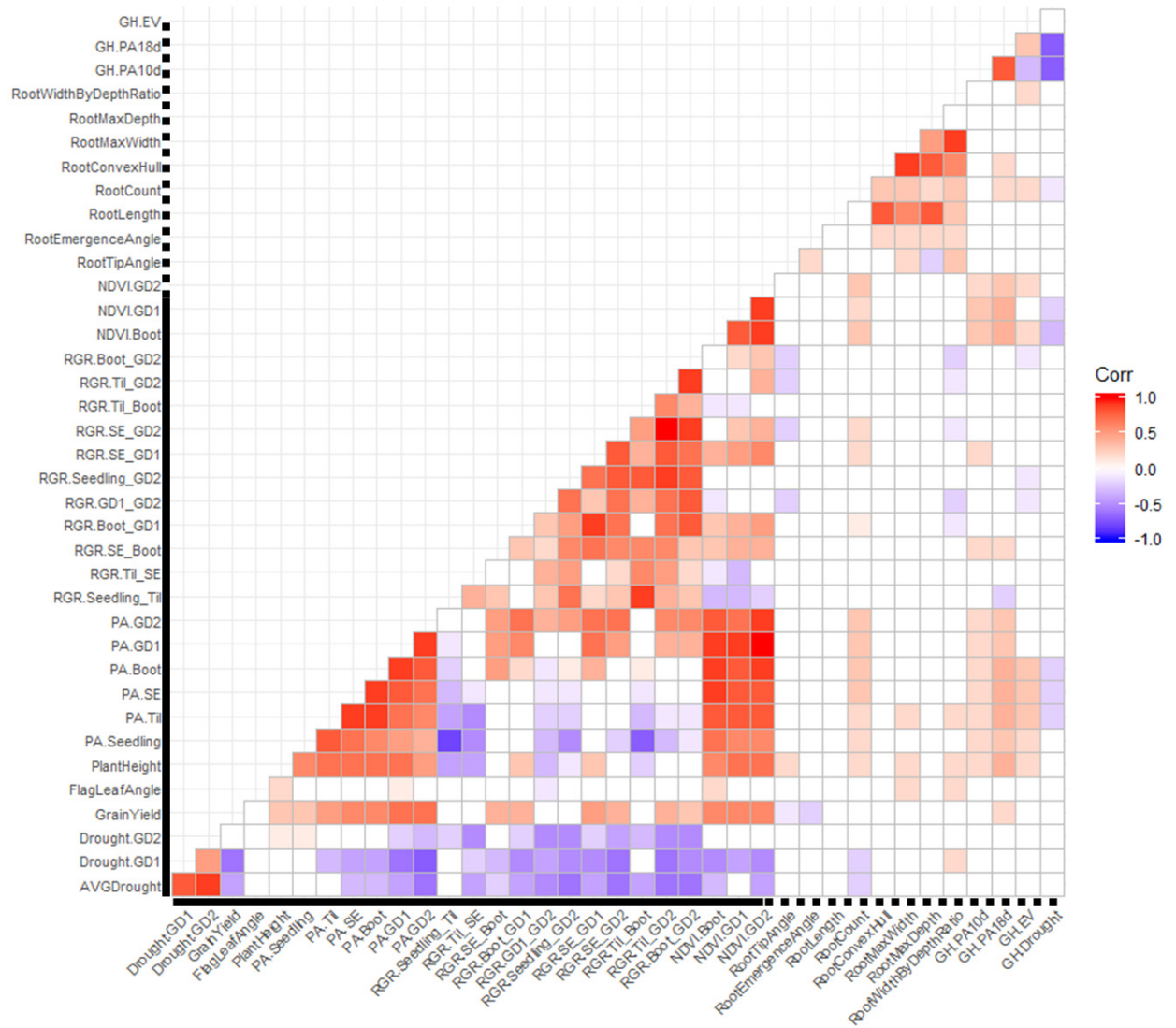

Figure 9. The correlation matrix of the traits measured in the field (solid lines) and under controlled conditions (dotted lines) in winter wheat. Filled boxes represent significant correlations ( $p$-value $<0.05)$ and are colored by the degree of correlation.

\subsection{Random Forest for Prediction of Drought Tolerance}

Drought scores obtained from average of scores obtained at GD1 and GD2 stages were predicted by using random forest models integrating all the phenotypic data obtained from the field and controlled conditions (Figure 10). Prediction of drought tolerance identified PA.GD2, RGR.Boot_GD2 RGR.GD1-GD2, NDVI.Boot, and RGR.Til_GD2 as top predictors. The RMSE and R2 of the model were 0.84 and 0.54 , respectively, with mtry 18 . Among the traits measured under the controlled conditions, GH.Drought was considered more important by the model followed by RootCount. Several other traits from the controlled conditions received low importance.

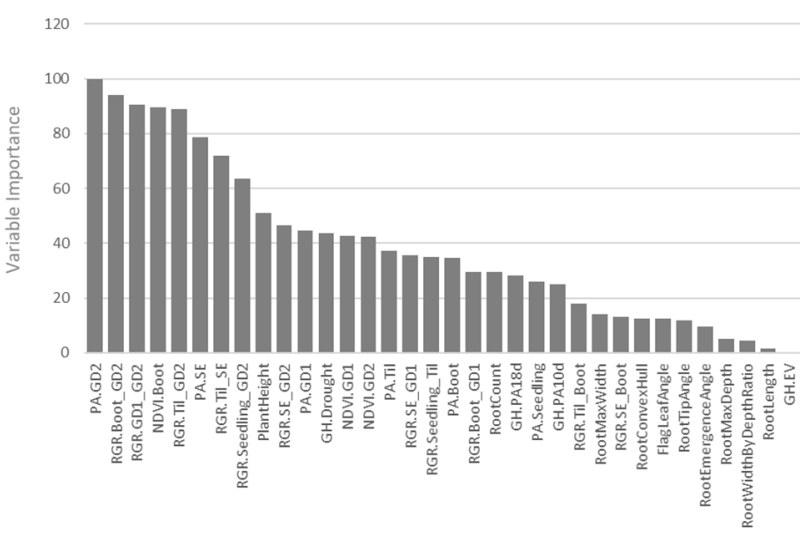

Figure 10. Variable importance from random forest predictions for drought tolerance. 


\section{Discussion}

A compilation of previous drought events in Europe from 1950 to 2012 identified 22 most prominent events in Europe [41]. Northern Europe and Russia were most affected by drought in the 1950s and 1960s [41]. Finland suffered severe drought from 1939-1942 followed by a below-average precipitation for the following three-and-a-half years [42]. Another drought in Finland in 2002-2003 occurred during the winter period, limiting the impact on agriculture [42]. The severe drought of 2018 in central and Northern Europe occurred during the peak growing season (Figure 11) and had a severe impact on the ecosystems [43] and high yield losses in winter wheat (Figure 12). Agricultural drought occurs due to low moisture content in the soil over a long period of time, negatively affecting crop production. The effect of drought on agriculture can be quantified using a drought index which is built from several different parameters such as soil moisture, precipitation, temperature, rainfall, and severity and duration of the same. Several drought indices have been proposed so far and reviewed previously by Mishra and Singh [44].

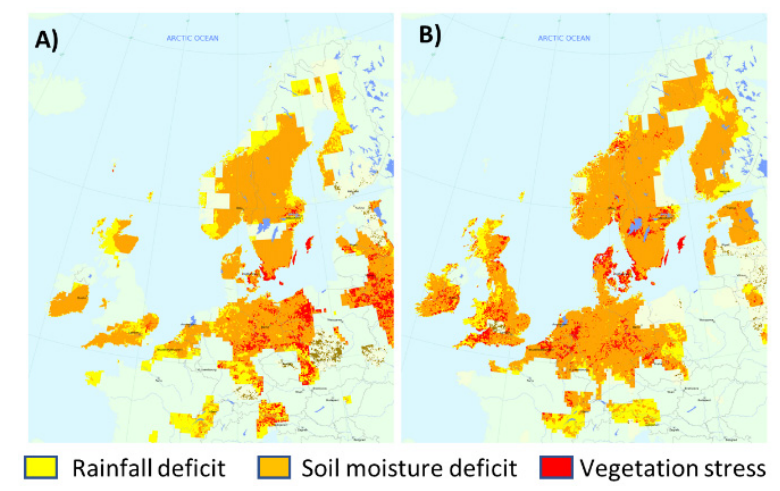

Figure 11. The drought indicator for Northern Europe during (A) June and (B) July 2018 based on estimations by the European drought observatory and generated using Copernicus Emergency Management Service information (2020) [45].

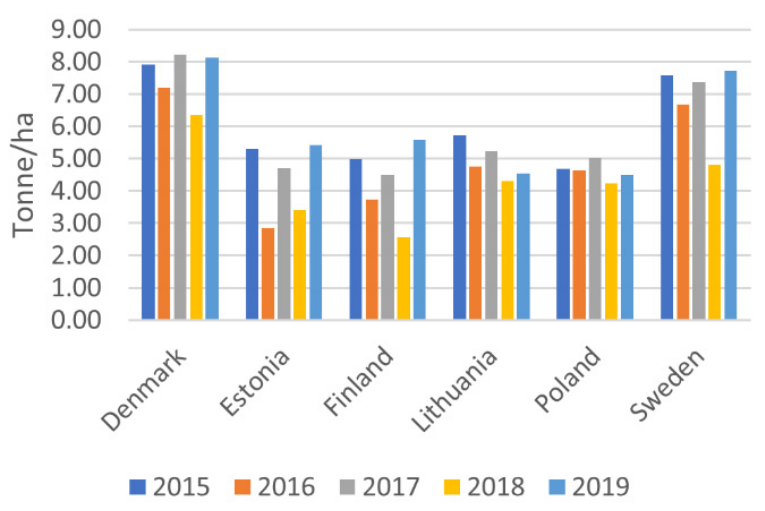

Figure 12. Winter wheat yields in six countries in the recent five years. Source: Eurostat.

There is a need for introgression of drought tolerance in the cultivars for Northern Europe. In this work, we characterized 142 winter wheat germplasm deposited at the genebank NordGen for drought tolerance both in the field and under controlled conditions. The germplasm studied in this work are old cultivars and landraces which are mainly of the Nordic origin and have previously been characterized for resistance to the disease septoria tritici blotch [46]. The observed variation for drought tolerance in the material indicates that this collection also has the potential to be used for introgressing drought tolerance in the elite winter wheat cultivars for Northern Europe. Sensor-based phenotyping has been used previously to study various morphological traits correlated with drought tolerance. Imaging-based plant biomass and RGR from the field correlated well with drought tolerance and can indicate early stress symptoms if the growth patterns differ from expected. It was earlier shown that RGR in early 
growth stages is positively correlated to drought tolerance in wheat under water deficit [15]. Simane, Peacock and Struik [16] found that the drought-tolerant genotypes had higher RGR in optimal conditions and low RGR in moisture stress, while the drought-susceptible genotypes showed the opposite trend. NDVI measurements also correlated with drought with the maximum correlation at the stem-elongation stage, thus making it a suitable proxy estimate for drought stress. NDVI was previously shown to be an effective indicator of plant response to drought stress $[17,18]$, and thus several QTL for NDVI were also identified [18]. Liu, Li, Zhou and Chen [19] defined a threshold for NDVI below which the plants were considered to be under drought stress. To evaluate if proxy measurements at the seedling stage can be used for selecting for drought tolerance, we characterized the germplasm for root and shoot growth at the seedling stage under controlled conditions. Root traits at the seedling stage such as root count and root tip angle moderately correlated with drought stress observed in the field. Deeper roots with more branching at depth are known to be more efficient at utilizing moisture from deeper soils [47]. However, the yield advantage of deeper roots is only seen in water-limited conditions [48]. Seedling root traits were earlier shown to correlate well with root traits at the vegetative stage in wheat but not at the reproductive stage [49]. In oats, moderate correlations were observed among root traits in the seedling stage grown in pots and rhizotrons and those in adult plants under field conditions [50]. Root traits are challenging and laborious to study under field conditions, and drought is unpredictable, making it difficult to select deep-rooted genotypes for drought tolerance. Faster, cheaper, and reliable assays are thus beneficial for efficient selection of germplasm for drought tolerance.

The traits studied in this work individually correlated moderately to adult plant drought stress in the field; however, the additive predictive power of these traits was harnessed using machine learning. The random forest models developed in this work utilized phenotypic measurements from both field and controlled conditions to predict adult plant drought stress levels in the field. NDVI, RGR, and plant biomass from the field and drought stress from the controlled conditions were selected as top predictors by random forest for drought stress. This is a promising approach to be able to select germplasm from the collective prediction power of traits obtained under different growth conditions and stages. The models developed in this work were validated with ten-fold cross-validation; however, an independent test set was not available. Thus, future work could involve a much larger data set for training and testing of machine learning models for drought stress under diverse environments. Machine learning approaches have been implemented earlier for identifying abiotic and biotic stresses in plants and is summarized previously [21,51,52]. However, previous studies on the use of machine learning for drought stress are scarce [51], and studying stem water potential in vineyards has been attempted [53,54]. Thus, such integrated approaches could further enable the integration of novel traits in accelerating the selection process in plant breeding.

Results from this work indicate that the consumer-grade cameras are cost-effective tools for automated phenotyping under field and controlled growth conditions. Plant biomass estimated by imaging with such cameras can help evaluate the underlying plant stress. Biomass estimated over time at several timepoints can help discern healthy plants from the stressed plants based on plant growth patterns. Results from random forest analysis in this work indicate that plant growth estimates obtained from digital cameras are top predictors for drought stress in plants. Thus, the use of digital cameras together with barebone phenotyping systems can make automated phenotyping affordable for wider use. In this work, outdoor phenotyping was mainly done by proximal phenotyping; however, for certain traits, sensor proximity to the plants is not as important as phenotyping at appropriate growth stages [54]. Unmanned aerial vehicles (UAVs) have also been used for studying drought stress in previous studies, which have been reviewed by Barbedo [51]. RGB, multispectral, and thermal imaging by UAVs have shown to be effective techniques for studying water stress deficit in plants [55]. UAVs equipped with RGB and NIR sensors are affordable alternatives for studying water deficit, and the effectiveness can be further improved by phenotyping plants several times during the plant growth cycle. 
In Northern Europe, meteorological drought is not a frequent occurrence, and thus drought tolerance is not a high-priority breeding target for cereal crops. Some of the secondary morphological traits leading to drought tolerance such as early vigour, RGR, and root architecture are also relevant for other traits such as nitrogen use efficiency [56,57] and phosphate uptake [58]. Thus, there is an added value in the characterization of germplasm for such secondary traits using affordable phenotyping. Therefore, a holistic breeding approach is required for breeding for new cultivars adapted to the changing climate by incorporating secondary traits beneficial for multiple primary traits of economical importance.

\section{Conclusions}

Phenocart mounted with consumer-grade digital cameras was evaluated in this work for characterizing winter wheat germplasm for drought tolerance. The results revealed that relative growth rates (RGR) of plants over the entire growth season are negatively correlated to drought tolerance. Root traits measured at seedling stage under controlled growth conditions were moderately positively correlated to drought tolerance under field conditions and can be integrated with the field-based metrics for evaluation of germplasm for drought tolerance. Random forest models were built by integrating data obtained from imaging under field and controlled growth conditions. Based on the results, it can be concluded that automated phenotyping systems built from low-cost equipment are a viable alternative which could facilitate broader acceptance of these systems. The drought-tolerant germplasm identified in this work can be used for introgressing drought tolerance in elite cultivars and for functional studies.

Supplementary Materials: The following are available online at http://www.mdpi.com/2073-4395/10/6/882/s1, Table S1: Manual scoring for drought tolerance at two grain development stages (GD1 and GD2). Table S2: Spearman's rank correlation coefficient analysis between traits.

Author Contributions: A.C. planned and designed the project. J.T.S. selected the germplasm. D.K. and A.C. performed the field experiments and analyzed field images. D.K. and C.D. performed the greenhouse experiment. T.H. performed the field trials in Sweden and G.B. and Ž.L. in Lithuania. D.K., S.K., C.D., V.V., and A.C. analyzed the data. D.K. and A.C. wrote the first draft. All authors have read and agreed to the published version of the manuscript.

Funding: This work was supported by funding from The Lantmännen Research Foundation (\#2018F001), SLU Grogrund (\#slu.ltv.2019.1.1.1-155), Nordic Council of Ministers (PPP \#6P2), NordForsk (\#84597), and Einar Nilssons Foundation.

Conflicts of Interest: The authors declare that they have no conflict of interest.

\section{References}

1. Shiferaw, B.; Smale, M.; Braun, H.-J.; Duveiller, E.; Reynolds, M.; Muricho, G. Crops that feed the world 10. Past successes and future challenges to the role played by wheat in global food security. Food Secur. 2013, 5, 291-317. [CrossRef]

2. Ray, D.K.; Mueller, N.D.; West, P.C.; Foley, J.A. Yield trends are insufficient to double global crop production by 2050. PLoS ONE 2013, 8, e66428. [CrossRef]

3. Tester, M.; Langridge, P. Breeding technologies to increase crop production in a changing world. Science 2010, 327, 818-822. [CrossRef] [PubMed]

4. Ceglar, A.; Toreti, A.; Lecerf, R.; Van der Velde, M.; Dentener, F. Impact of meteorological drivers on regional inter-annual crop yield variability in France. Agric. For. Meteorol. 2016, 216, 58-67. [CrossRef]

5. Olesen, J.E.; Jensen, T.; Petersen, J. Sensitivity of field-scale winter wheat production in Denmark to climate variability and climate change. Clim. Res. 2000, 15, 221-238. [CrossRef]

6. Chawade, A.; Armoniené, R.; Berg, G.; Brazauskas, G.; Frostgård, G.; Geleta, M.; Gorash, A.; Henriksson, T.; Himanen, K.; Ingver, A. A transnational and holistic breeding approach is needed for sustainable wheat production in the Baltic Sea region. Physiol. Plant. 2018, 164, 442-451. [CrossRef] [PubMed]

7. Team, B.A. Second assessment of climate change for the Baltic Sea basin. In Regional Climate Studies; Springer: Berlin/Heidelberg, Germany, 2015; Volume 6, pp. 131-144. 
8. Chenu, K.; Deihimfard, R.; Chapman, S.C. Large-scale characterization of drought pattern: A continent-wide modelling approach applied to the Australian wheatbelt-spatial and temporal trends. New Phytol. 2013, 198, 801-820. [CrossRef] [PubMed]

9. Furbank, R.T.; Tester, M. Phenomics-Technologies to relieve the phenotyping bottleneck. Trends Plant Sci. 2011, 16, 635-644. [CrossRef]

10. McCouch, S.; Baute, G.J.; Bradeen, J.; Bramel, P.; Bretting, P.K.; Buckler, E.; Burke, J.M.; Charest, D.; Cloutier, S.; Cole, G. Agriculture: Feeding the future. Nature 2013, 499, 23. [CrossRef]

11. White, J.W.; Conley, M.M. A flexible, low-cost cart for proximal sensing. Crop Sci. 2013, 53, 1646-1649. [CrossRef]

12. Virlet, N.; Sabermanesh, K.; Sadeghi-Tehran, P.; Hawkesford, M.J. Field Scanalyzer: An automated robotic field phenotyping platform for detailed crop monitoring. Funct. Plant Biol. 2017, 44, 143-153. [CrossRef] [PubMed]

13. Rutkoski, J.; Poland, J.; Mondal, S.; Autrique, E.; Pérez, L.G.; Crossa, J.; Reynolds, M.; Singh, R. Canopy temperature and vegetation indices from high-throughput phenotyping improve accuracy of pedigree and genomic selection for grain yield in wheat. G3 Genes Genomes Genet. 2016, 6, 2799-2808. [CrossRef] [PubMed]

14. Thorp, K.R.; Gore, M.; Andrade-Sanchez, P.; Carmo-Silva, A.; Welch, S.; White, J.; French, A.N. Proximal hyperspectral sensing and data analysis approaches for field-based plant phenomics. Comput. Electron. Agric. 2015, 118, 225-236. [CrossRef]

15. Solomon, K.F.; Labuschagne, M.T. Morpho-physiological response of durum wheat genotypes to drought stress. S. Afr. J. Plant Soil 2013, 26, 141-146. [CrossRef]

16. Simane, B.; Peacock, J.M.; Struik, P.C. Differences in developmental plasticity and growth rate among drought-resistant and susceptible cultivars of durum wheat (Triticum turgidum L. var. durum). Plant Soil 1993, 157, 155-166. [CrossRef]

17. Reynolds, M.; Dreccer, F.; Trethowan, R. Drought-adaptive traits derived from wheat wild relatives and landraces. J. Exp. Bot. 2006, 58, 177-186. [CrossRef]

18. Condorelli, G.E.; Maccaferri, M.; Newcomb, M.; Andrade-Sanchez, P.; White, J.W.; French, A.N.; Sciara, G.; Ward, R.; Tuberosa, R. Comparative Aerial and Ground Based High Throughput Phenotyping for the Genetic Dissection of NDVI as a Proxy for Drought Adaptive Traits in Durum Wheat. Front. Plant Sci. 2018, 9. [CrossRef]

19. Liu, Z.; Li, C.; Zhou, P.; Chen, X. A probabilistic assessment of the likelihood of vegetation drought under varying climate conditions across China. Sci. Rep. 2016, 6. [CrossRef]

20. An, J.; Li, W.; Li, M.; Cui, S.; Yue, H. Identification and Classification of Maize Drought Stress Using Deep Convolutional Neural Network. Symmetry 2019, 11, 256. [CrossRef]

21. Singh, A.; Ganapathysubramanian, B.; Singh, A.K.; Sarkar, S. Machine Learning for High-Throughput Stress Phenotyping in Plants. Trends Plant Sci. 2016, 21, 110-124. [CrossRef]

22. Aghabozorgi, S.; Shirkhorshidi, A.S.; Wah, T.Y. Time-series clustering-A decade review. Inf. Syst. 2015, 53, 16-38. [CrossRef]

23. Montes, J.; Technow, F.; Dhillon, B.; Mauch, F.; Melchinger, A. High-throughput non-destructive biomass determination during early plant development in maize under field conditions. Field Crops Res. 2011, 121, 268-273. [CrossRef]

24. Paparrizos, J.; Gravano, L. k-Shape: Efficient and Accurate Clustering of Time Series. In Proceedings of the 2015 ACM SIGMOD International Conference on Management of Data, Melbourne, Australia, 31 May-4 June 2015; pp. 1855-1870.

25. Gracia-Romero, A.; Kefauver, S.C.; Fernandez-Gallego, J.A.; Vergara-Díaz, O.; Nieto-Taladriz, M.T.; Araus, J.L. UAV and Ground Image-Based Phenotyping: A Proof of Concept with Durum Wheat. Remote Sens. 2019, 11, 1244. [CrossRef]

26. Gracia-Romero, A.; Vergara-Díaz, O.; Thierfelder, C.; Cairns, J.; Kefauver, S.; Araus, J. Phenotyping conservation agriculture management effects on ground and aerial remote sensing assessments of maize hybrids performance in Zimbabwe. Remote Sens. 2018, 10, 349. [CrossRef]

27. Buchaillot, M.; Gracia-Romero, A.; Vergara-Diaz, O.; Zaman-Allah, M.A.; Tarekegne, A.; Cairns, J.E.; Prasanna, B.M.; Araus, J.L.; Kefauver, S.C. Evaluating Maize Genotype Performance under Low Nitrogen Conditions Using RGB UAV Phenotyping Techniques. Sensors 2019, 19, 1815. [CrossRef] 
28. Tripodi, P.; Massa, D.; Venezia, A.; Cardi, T. Sensing technologies for precision phenotyping in vegetable crops: Current status and future challenges. Agronomy 2018, 8, 57. [CrossRef]

29. Crain, J.L.; Wei, Y.; Barker, J.; Thompson, S.M.; Alderman, P.D.; Reynolds, M.; Zhang, N.; Poland, J. Development and Deployment of a Portable Field Phenotyping Platform. Crop Sci. 2016, 56. [CrossRef]

30. Zadoks, J.C.; Chang, T.T.; Konzak, C.F. A decimal code for the growth stages of cereals. Weed Res. 1974, 14, 415-421. [CrossRef]

31. IRRI. Standard Evaluation System for Rice (SES), 5th ed.; International Rice Research Institute (IRRI): Los Baños, Philippines, 2013.

32. Armoniené, R.; Odilbekov, F.; Vivekanand, V.; Chawade, A. Affordable imaging lab for noninvasive analysis of biomass and early vigour in cereal crops. BioMed Res. Int. 2018, 2018, 9. [CrossRef]

33. Thomas, C.L.; Graham, N.S.; Hayden, R.; Meacham, M.C.; Neugebauer, K.; Nightingale, M.; Dupuy, L.X.; Hammond, J.P.; White, P.J.; Broadley, M.R. High-throughput phenotyping (HTP) identifies seedling root traits linked to variation in seed yield and nutrient capture in field-grown oilseed rape (Brassica napus L.). Ann. Bot. 2016, 118, 655-665. [CrossRef]

34. DigiCamControl. Available online: http://digicamcontrol.com/ (accessed on 1 March 2019).

35. Rawtherapee. Available online: https://rawtherapee.com/ (accessed on 1 February 2019).

36. Gehan, M.A.; Fahlgren, N.; Abbasi, A.; Berry, J.C.; Callen, S.T.; Chavez, L.; Doust, A.N.; Feldman, M.J.; Gilbert, K.B.; Hodge, J.G. PlantCV v2: Image analysis software for high-throughput plant phenotyping. PeerJ 2017, 5, e4088. [CrossRef] [PubMed]

37. Pound, M.P.; French, A.P.; Atkinson, J.A.; Wells, D.M.; Bennett, M.J.; Pridmore, T. RootNav: Navigating images of complex root architectures. Plant Physiol. 2013, 162, 1802-1814. [CrossRef] [PubMed]

38. Mendiburu, F.D. Agricolae: Statistical Procedures for Agricultural Research, R package version 1.2-8. 2017.

39. R Core Team. R: A Language and Environment for Statistical Computing; R Foundation for Statistical Computing: Vienna, Austria, 2017.

40. Hunt, R. Plant Growth Curves: The Functional Approach to Plant Growth Analysis; Arnold: London, UK, 1982; Volume viii, p. 248.

41. Spinoni, J.; Naumann, G.; Vogt, J.V.; Barbosa, P. The biggest drought events in Europe from 1950 to 2012. J. Hydrol. Reg. Stud. 2015, 3, 509-524. [CrossRef]

42. Veijalainen, N.; Ahopelto, L.; Marttunen, M.; Jääskeläinen, J.; Britschgi, R.; Orvomaa, M.; Belinskij, A.; Keskinen, M. Severe Drought in Finland: Modeling Effects on Water Resources and Assessing Climate Change Impacts. Sustainability 2019, 11, 2450. [CrossRef]

43. Buras, A.; Rammig, A.; Zang, C.S. Quantifying impacts of the drought 2018 on European ecosystems in comparison to 2003. Biogeosciences 2019. [CrossRef]

44. Mishra, A.K.; Singh, V.P. A review of drought concepts. J. Hydrol. 2010, 391, 202-216. [CrossRef]

45. European Drought Observatory. Available online: https://edo.jrc.ec.europa.eu/edov2 (accessed on 29 January 2020).

46. Odilbekov, F.; Armoniené, R.; Koc, A.; Svensson, J.; Chawade, A. GWAS-Assisted Genomic Prediction to Predict Resistance to Septoria Tritici Blotch in Nordic Winter Wheat at Seedling Stage. Front. Genet. 2019, 10. [CrossRef]

47. Manschadi, A.M.; Christopher, J.; deVoil, P.; Hammer, G.L. The role of root architectural traits in adaptation of wheat to water-limited environments. Funct. Plant Biol. 2006, 33. [CrossRef]

48. El Hassouni, K.; Alahmad, S.; Belkadi, B.; Filali-Maltouf, A.; Hickey, L.T.; Bassi, F.M. Root System Architecture and Its Association with Yield under Different Water Regimes in Durum Wheat. Crop Sci. 2018, 58, 2331-2346. [CrossRef]

49. Watt, M.; Moosavi, S.; Cunningham, S.C.; Kirkegaard, J.A.; Rebetzke, G.J.; Richards, R.A. A rapid, controlled-environment seedling root screen for wheat correlates well with rooting depths at vegetative, but not reproductive, stages at two field sites. Ann. Bot. 2013, 112, 447-455. [CrossRef]

50. Canales, F.J.; Nagel, K.A.; Müller, C.; Rispail, N.; Prats, E. Deciphering Root Architectural Traits Involved to Cope With Water Deficit in Oat. Front. Plant Sci. 2019, 10. [CrossRef] [PubMed]

51. Barbedo, J.G.A. A Review on the Use of Unmanned Aerial Vehicles and Imaging Sensors for Monitoring and Assessing Plant Stresses. Drones 2019, 3, 40. [CrossRef]

52. Humplík, J.F.; Lazár, D.; Husičková, A.; Spíchal, L. Automated phenotyping of plant shoots using imaging methods for analysis of plant stress responses-A review. Plant Methods 2015, 11. [CrossRef] [PubMed] 
53. Poblete, T.; Ortega-Farías, S.; Moreno, M.; Bardeen, M. Artificial Neural Network to Predict Vine Water Status Spatial Variability Using Multispectral Information Obtained from an Unmanned Aerial Vehicle (UAV). Sensors 2017, 17, 2488. [CrossRef] [PubMed]

54. Romero, M.; Luo, Y.; Su, B.; Fuentes, S. Vineyard water status estimation using multispectral imagery from an $\mathrm{UAV}$ platform and machine learning algorithms for irrigation scheduling management. Comput. Electron. Agric. 2018, 147, 109-117. [CrossRef]

55. Berger, B.; Parent, B.; Tester, M. High-throughput shoot imaging to study drought responses. J. Exp. Bot. 2010, 61, 3519-3528. [CrossRef]

56. Garnett, T.; Conn, V.; Kaiser, B.N. Root based approaches to improving nitrogen use efficiency in plants. Plant Cell Environ. 2009, 32, 1272-1283. [CrossRef]

57. Tuberosa, R. Phenotyping for drought tolerance of crops in the genomics era. Front. Physiol. 2012, 3. [CrossRef]

58. Ryan, P.R.; Liao, M.; Delhaize, E.; Rebetzke, G.J.; Weligama, C.; Spielmeyer, W.; James, R.A. Early vigour improves phosphate uptake in wheat. J. Exp. Bot. 2015, 66, 7089-7100. [CrossRef]

(C) 2020 by the authors. Licensee MDPI, Basel, Switzerland. This article is an open access article distributed under the terms and conditions of the Creative Commons Attribution (CC BY) license (http://creativecommons.org/licenses/by/4.0/). 